\title{
Working with simple machines
}

\author{
John W. Norbury \\ Department of Physics and Astronomy, University of Southern Mississippi, \\ Hattiesburg, Mississippi, 39402, USA \\ E-mail: john.norbury@usm.edu
}

\begin{abstract}
A set of examples is provided that illustrate the use of work as applied to simple machines. The ramp, pulley, lever and hydraulic press are common experiences in the life of a student and their theoretical analysis therefore makes the abstract concept of work more real. The mechanical advantage of each of these systems is also discussed so that students can evaluate their usefulness as machines.
\end{abstract}




\section{Introduction}

The Work-Energy theorem (discussed in detail below) is one of the most important ideas in classical mechanics, and is often discussed in high school physics courses and university level freshman physics classes. Nevertheless, the reason for defining work as force times distance often remains obscure to the student. And the idea that work is conserved (in the absence of dissipative forces), in that a smaller force implies that the distance must be larger in order to obtain the same work (and thus impart the same energy to an object), is often lost on students. On the other hand, simple machines are often studied in elementary school science classes. These students get to experience how machines can amplify forces. For instance with a simple lever, the students can lift weights that would otherwise be impossible. Usually what is emphasized in the study of simple machines is the idea of mechanical advantage, or force amplification. This is fine and students learn from it. However rarely is the idea of work emphasized in the study of simple machines. The idea of the present article is to emphasize the constancy of work in the use of simple machines. The aim is twofold. Firstly, it is hoped that after going through the exercises below, high school and university level physics students will have a much clearer understanding as to why work is defined as force times distance. Simple machines will be used to illustrate the definition of work. Secondly, it is hoped that in the study of simple machines in elementary school, more attention will be paid to the idea of work. Work will be used to illustrate the use of simple machines.

The outline of the article is as follows. Four different types of simple machines (ramp, pulley, lever, hydraulic press) will be studied. In each case it will be shown explicitly that if less force is applied then a corresponding greater moving distance is involved such that the work (or effort) always remains the same, and correspondingly therefore the energy imparted is the same. The theme will be less force, more distance, same work. (Or if you like one can say more force, less distance, same work.) A good set of references on simple machines is provided [1, 2, 3, 4, 15, 6, 17, 8, 9, 10, 11, 12, One of the best is the book by Lehrman [4]. There are also some good sources for elementary school teachers [1, 2, 3] and high school and university teachers [4, 5, 6, 17, 8, 9, 10, 11, 12].

\subsection{Work-Energy theorem}

The force approach to the study of mechanics is to identify all the forces, divide by mass to get acceleration and then solve for velocity, displacement, time, etc. There is an alternative formulation of mechanics which does not rely heavily on force, but rather is based on the concepts of work and energy. The work-energy formulation of mechanics [13] is worthwhile since sometimes it is easier to use and involves only scalar quantities. Also it leads to a better physical understanding of mechanics. However the key reason for introducing work-energy is because energy is conserved. This great discovery simplified a great deal of physics. The basic concept of work is that it is force times distance. You do work on an object by applying a force over a certain distance. When you lift an object you apply a lifting force over the height that you lift the object. 
Machines are devices that allow us to do work more efficiently. For example, a ramp is what is called a simple machine. If you load objects into a truck, then a large ramp (large distance) allows you to apply less force to achieve the same work. Actually the proper physical definition of work is more complicated than simply force times distance. The proper definition is $W \equiv \int_{r_{i}}^{r_{f}} \mathbf{F} \cdot d \mathbf{r}$. Consider the 1-dimensional case. If the force $F_{x}$ is constant then it can be taken outside the integral to give

$$
W \equiv F_{x} \int_{x_{i}}^{x_{f}} d x=F_{x} \Delta x=\text { force } \times \text { distance }
$$

where $\Delta x \equiv x_{f}-x_{i}$. Using $\mathbf{F}=m \mathbf{a}$, and in the 1-dimensional case

$$
W=m \int_{x_{i}}^{x_{f}} a d x=m \int_{x_{i}}^{x_{f}} v \frac{d v}{d x} d x=\frac{1}{2} m v_{f}^{2}-\frac{1}{2} m v_{i}^{2} \equiv \Delta K
$$

where Kinetic Energy is defined as $K \equiv \frac{1}{2} m v^{2}$. Thus the total work is always equal to the change in kinetic energy. Now recognize that there are two types of forces called conservative and non-conservative. To put it briefly, conservative forces "bounce back" and non-conservative forces don't. Gravity is a conservative force. If you lift an object against gravity and let it go then the object falls back to where it began. Spring forces are conservative. If you pull a spring and then let it go, it bounces back to where it was. However friction is non-conservative. If you slide an object along the table against friction and let go, then the object just stays there. With conservative forces we always associate a potential energy. Thus any force $\mathbf{F}$ can be broken up into the conservative piece $\mathbf{F}_{C}$ and the non-conservative piece $\mathbf{F}_{N C}$, as in

$$
W \equiv \int_{r_{i}}^{r_{f}} \mathbf{F} \cdot d \mathbf{r}=\int_{r_{i}}^{r_{f}} \mathbf{F}_{C} \cdot d \mathbf{r}+\int_{r_{i}}^{r_{f}} \mathbf{F}_{N C} \cdot d \mathbf{r} \equiv W_{C}+W_{N C}
$$

and each piece corresponds therefore to conservative work $W_{C}$ and non-conservative work $W_{N C}$. Define the conservative piece as the negative of the change in a new quantity called potential energy $U$ and $W_{C} \equiv-\Delta U$, giving the Work-Energy theorem,

$$
\Delta U+\Delta K=W_{N C}
$$

If the non-conservative work is zero then we have conservation of total mechanical energy $\Delta E=0$, with $E \equiv U+K$.

\section{Ramp}

The ramp (or inclined plane) is shown in Figure 1, where $h$ is the height of the ramp and $s$ is the distance along the ramp. A weight exerts a vertical force $m g$. For an elementary science class two ramps can be constructed in which the distance $s$ of one ramp is double the distance of the other. However both ramps should have the same vertical distance $h$. An example might be loading furniture into a truck and choosing a ramp to make the job easier. Students can be asked to push the weight up both ramps. (It is essential to put the weight on wheels so that friction is minimized.) Thus the students will be pushing the weight through the distance $s$. The force that the students will have to overcome is then $m g \sin \theta$. See Figure 1. 


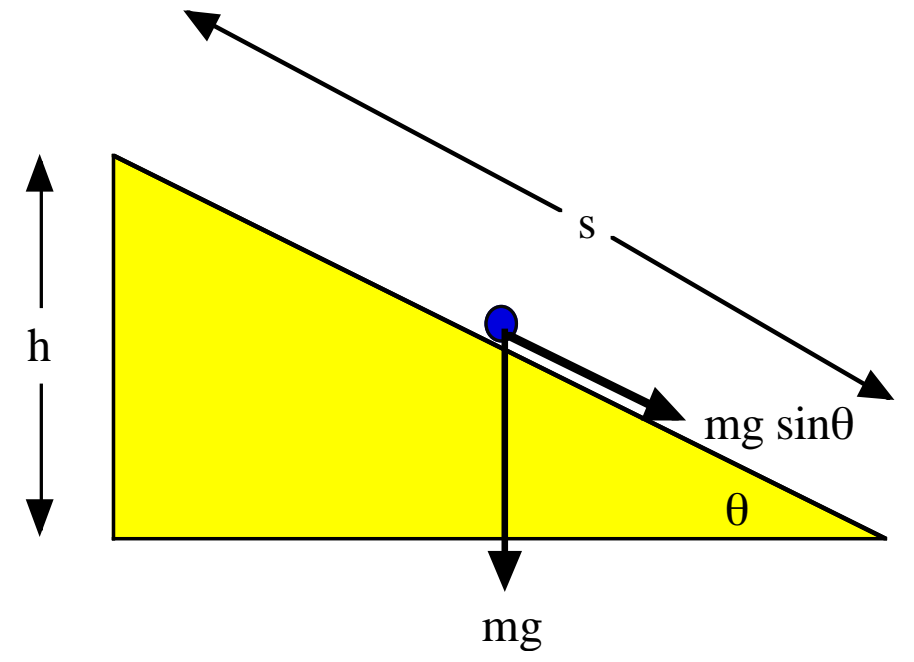

Figure 1. Ramp.

The essential idea for elementary school students to understand is the following. For a long ramp students will notice that the weight is easier to push. Nevertheless they will be pushing over a correspondingly greater distance and at the end of pushing the weight up both ramps, they will have expended the same effort. That is their work is the same. The final potential energy $(m g h)$ of the weight is the same. Less force, more distance, same work.

High school and university students can also work this result out mathematically as follows. The distance up the ramp is

$$
s=\frac{h}{\sin \theta}
$$

which is large for small angles. The pushing force (see Figure 1) is

$$
F=m g \sin \theta
$$

which is small for small angles. Thus ramps are easy to use when the angle is small. The product of force and distance is

$$
F s=m g \sin \theta \frac{h}{\sin \theta}=m g h .
$$

Thus for both ramps the product $F s$ is the same. This clearly shows that you can't get something for nothing. Less force, more distance, same work. Students should now have a clear grasp as to why the work (or effort) is defined as force times distance. It makes sense with the intuitive idea experienced in actually pushing the weight up the two different ramps. The effort expended in both cases was the same and this is embodied in the definition of work. (Note that of course work is really defined as the integral of the scalar product of force and displacement. This complication is not discussed in this article as the main idea is to develop intuition about the concept of work.) Most books which discuss simple machines emphasize mechanical advantage rather than work. For completeness, the mechanical advantage (MA) is defined as the load force divided by the input force or

$$
\mathrm{MA} \equiv \frac{\text { Load force }}{\text { Input force }}=\frac{m g}{m g \sin \theta}=\frac{1}{\sin \theta}=\frac{s}{h} .
$$


Thus a long ramp (large $s$ ) gives a large mechanical advantage.
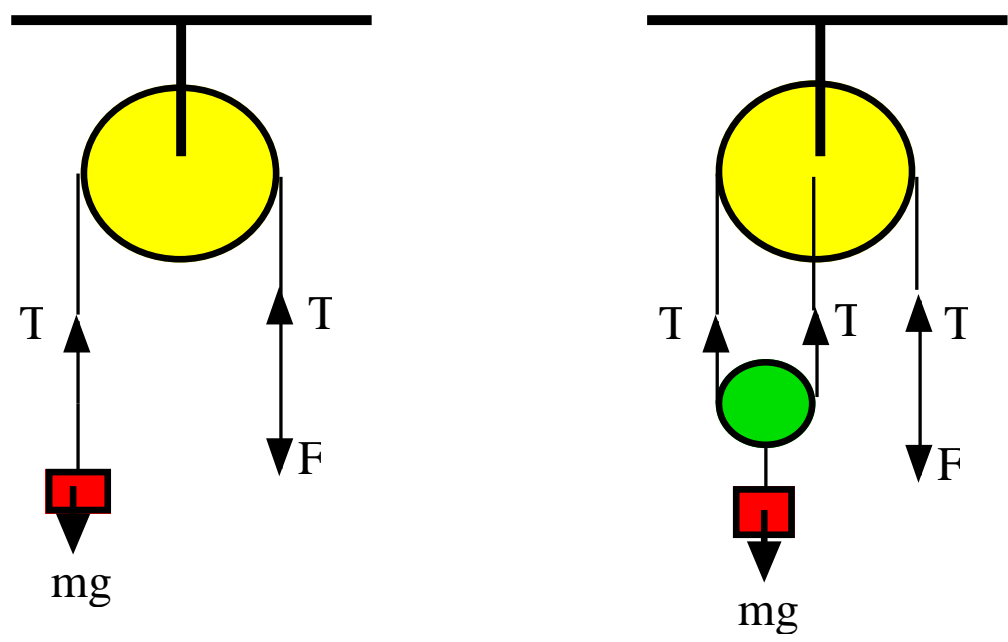

Figure 2. Pulley Systems.

\section{Pulley}

Next consider some pulley systems as shown in Figure 2. For simplicity only consider a single and double pulley. These will be sufficient to illustrate the main idea. Imagine that the weight is pulled with a force $F$ at constant speed so that the acceleration is zero. Applying $F=m a$ to the weight gives $T-m g=0$ where $T$ is the tension. Thus the pulling force $F$ equals $T$ and

$$
F=m g .
$$

The rope in the pulley is pulled through a distance $s$. If the weight is to be raised by a height $h$ then obviously

$$
s=h .
$$

Now consider the two pulley system in Figure 2. Again applying $F=m a$ to the weight gives $2 T-m g=0$ or $T=\frac{m g}{2}$ giving

$$
F=\frac{m g}{2} \text {. }
$$

Now by looking at Figure 2 it can be seen that if the rope is pulled through a distance of $s$ then the weight will only be raised by $h=\frac{s}{2}$. Thus now

$$
s=2 h \text {. }
$$

Thus for the double pulley, it's twice as easy to lift the weight, but one has to pull double the distance to raise the weight the same height. By actually using these pulley systems the students can experience this for themselves.

The product of force and distance however remains the same.

$$
F s=\frac{m g}{2} 2 h=m g h
$$


Less force, more distance, same work. Elementary school students can simply experience this for themselves by using the two pulley systems. High school and university students can do the calculations to show explicitly that the product $F s$ remains the same and thus is good for a definition of work.

The mechanical advantage is

$$
\mathrm{MA} \equiv \frac{\text { Load force }}{\text { Input force }}=\frac{m g}{m g / 2}=2=\frac{s}{h}
$$



Figure 3. Lever.

\section{Lever}

The lever is another famous simple machine and is shown in Figure 3. In the top part of the figure, a lever is shown in the horizontal position and one imagines that a person is pushing down with the force $F$ to hold a weight $m g$ in balance. This will be the case no matter what is the orientation of the lever. We will ignore the weight of the lever and imagine that we have a lever made of a very light material that does not bend. For static equilibrium the torques produced by both forces must be the same, $\tau_{1}=\tau_{2}$ giving $m g a=F b$ or

$$
F=m g \frac{a}{b}
$$

Thus only a small pushing force $F$ is needed if the lever arm $b$ is large. This is something easily demonstrated to students. However if one is to use the lever to lift the weight, then the longer the lever arm, the larger the distance $s$ one will have to lift through. This is seen in the bottom part of Figure 3. From the figure $\theta=\frac{h}{a}=\frac{s}{b}$ where $h$ is the distance the object is to be lifted. The distance that the lever arm will have to be moved through is

$$
s=h \frac{b}{a}
$$


showing that if the lever arm $b$ is large (small force) the distance $s$ must be large. All students can experience this using the lever. The point is though that the product of force and distance will always be the same,

$$
F s=m g \frac{a}{b} h \frac{b}{a}=m g h .
$$

Once again, elementary school students can use the lever, change the length of the lever arm and notice that even though it's easier to lift, they have to lift through a larger distance $s$ and their work, or effort, remains the same. High school and university students can demonstrate mathematically that the work remains the same. Again we have the principle of less force, more distance, same work.

The mechanical advantage is

$$
\mathrm{MA} \equiv \frac{\text { Load force }}{\text { Input force }}=\frac{m g}{F}=\frac{b}{a}=\frac{s}{h} .
$$

\section{Hydraulic Press}

Finally the hydraulic press can also be used to demonstrate these ideas. The press is shown in Figure 4. The pressure $P$ is defined as

$$
\text { Pressure } \equiv \frac{\text { Force }}{\text { Area }}
$$

but the pressure throughout the fluid is the same. Thus

$$
P=\frac{F}{A_{1}}=\frac{m g}{A_{2}}
$$

giving the applied force as

$$
F=\frac{A_{1}}{A_{2}} m g
$$

which shows that for a large area $A_{2}$ then only a small force $F$ need be applied. However from Figure 4 it can be seen that if $A_{2}$ is large then the weight $m g$ will only be lifted a

small distance $h$. This can be seen mathematically. The volume change $\Delta V$ will be the same,

$$
\Delta V=A_{1} s=A_{2} h
$$

giving

$$
s=\frac{A_{2}}{A_{1}} h
$$

showing that if $A_{2}$ is large (small force needed) then the distance $s$ over which the force must be applied will have to be large. Students can experience this by using two different hydraulic presses with different areas $A_{2}$. The work is always the same. Mathematically this is seen from

$$
F s=\frac{A_{1}}{A_{2}} m g \frac{A_{2}}{A_{1}} h=m g h
$$


For the hydraulic press less force, more distance, same work. The mechanical advantage is

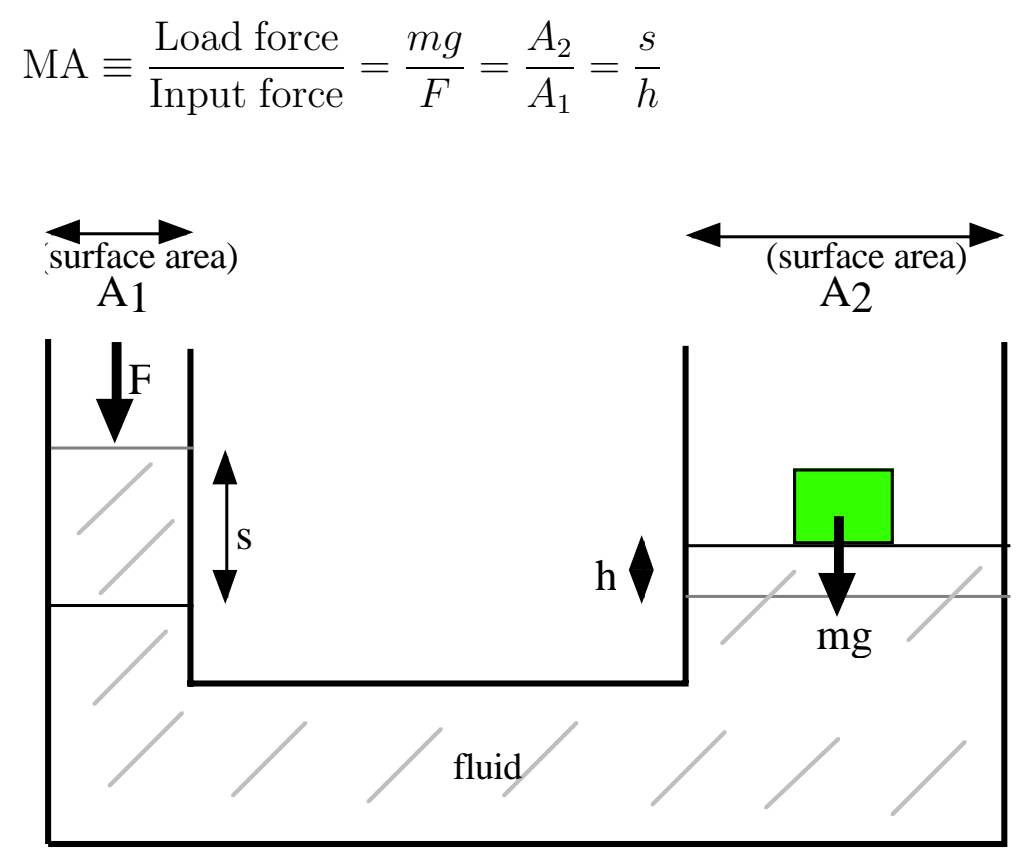

Figure 4. Hydraulic Press.

In this section we have assumed an idealised hydraulic press. However in reality frictional effects can be rather large and this will make the mechanical advantage smaller than the ideal case.

\section{Conclusion}

The simple machines consisting of a ramp, pulley, lever and hydraulic press are found in everyday life. Their mathematical analysis makes the concept of work much clearer to students encountering the subject for the first time. These machines can be easily constructed and used as demonstrations in the classroom, which makes them doubly effective for learning. When students interact with these demonstrations they can easily experience the concept of mechanical advantage which has been presented in this paper. Both demonstrations and the analysis of this paper, make simple machines an excellent way for students to learn about work.

\section{References}

[1] Carin A A 1997 Guided discovery activities for elementary school science (New Jersey: PrenticeHall) pp A-53 - A-66

[2] Harlin J D and Rivkin M S 1996 Science experiences for the early childhood years (New Jersey: Prentice-Hall) pp 223 - 239

[3] National Academy Press 1996 Resources for teaching elementary school science (Washington D.C.: National Academy Press) pp 68 -95

[4] Lehrman R L 1990 Physics the easy way (New York: Barron's Educational Series) pp 95 - 117 
[5] Harris N C and Hemmerling E M 1963 Introductory applied physics 2nd ed (New York: McGrawHill) pp 181 - 210

[6] Beiser A 1995 Applied Physics 3rd ed (New York: McGraw-Hill) pp 147 - 158

[7] Bueche F J 1997 College Physics 8th ed (New York: McGraw-Hill) pp 68 - 73

[8] Halpern A 1995 Beginning Physics I: mechanics and heat (New York: McGraw-Hill) pp 172 - 188

[9] Hooper H O and Gwynne P 1980 Physics and the physical perspective 2nd ed (San Francisco: Harper and Row) pp 136 - 139

[10] Lea S M and Burke J R 1997 Physics: the nature of things (New York: West) pp 237 - 239

[11] Fishbane P M, Gasiorowicz S and Thornton S T 1996 Physics for scientists and engineers 2nd ed (New Jersey: Prentice-Hall) pp 117 - 149

[12] Hecht E 1996 Physics calculus (Pacific Grove, California: Brooks/Cole) pp 406 - 408

[13] Serway R A and Jewett J W 2002 Principles of Physics 3rd ed, vol 1 (New York: Harcourt College Publishers). 